PROCEEDINGS OF THE

AMERICAN MATHEMATICAL SOCIETY

Volume 131, Number 2, Pages 371-377

$\mathrm{S}$ 0002-9939(02)06593-0

Article electronically published on June 3, 2002

\title{
FULLY TRANSITIVE $p$-GROUPS WITH FINITE FIRST ULM SUBGROUP
}

\author{
AGNES T. PARAS AND LUTZ STRÜNGMANN
}

(Communicated by Stephen D. Smith)

\begin{abstract}
An abelian $p$-group $G$ is called (fully) transitive if for all $x, y \in G$ with $U_{G}(x)=U_{G}(y)\left(U_{G}(x) \leq U_{G}(y)\right)$ there exists an automorphism (endomorphism) of $G$ which maps $x$ onto $y$. It is a long-standing problem of A. L. S. Corner whether there exist non-transitive but fully transitive $p$-groups with finite first Ulm subgroup. In this paper we restrict ourselves to $p$-groups of type $A$, this is to say $p$-groups satisfying $\operatorname{Aut}(G) \uparrow_{p^{\omega} G}=U\left(\operatorname{End}(G) \uparrow_{p}{ }^{\omega} G\right)$. We show that the answer to Corner's question is no if $p^{\omega} G$ is finite and $G$ is of type $A$.
\end{abstract}

\section{INTRODUCTION}

The notions of transitivity and full transitivity originated in the book "Infinite Abelian Groups" by I. Kaplansky 11] and extensive classes of abelian p-groups which are both transitive and fully transitive were found in [8] and [10]. Moreover, $p$-groups with neither property were constructed in [13] and for larger cardinalities in [7. In [5] Files and Goldsmith proved the surprising result that a $p$-group $G$ is fully transitive if and only if its square $G \oplus G$ is transitive. Nevertheless, for $p=2$ the independence of both concepts was shown by Corner in 4 and already Kaplansky had shown in 11 that for $p>2$ transitivity always implies full transitivity. Therefore it is natural to ask which fully transitive non-transitive $p$-groups appear. By a fundamental observation of Corner 4] one can reduce the decision of whether or not a $p$-group $G$ is (fully) transitive to its first Ulm subgroup $p^{\omega} G$. In [4] it was shown that $G$ is (fully) transitive if and only if $\operatorname{End}(G)\left\lceil p^{\omega} G\right.$ acts (fully) transitively on the first Ulm subgroup $p^{\omega} G$ of $G$, i.e., for any $x, y \in p^{\omega} G$ such that $U_{p^{\omega} G}(x)=U_{p^{\omega} G}(y)\left(U_{p^{\omega} G}(x) \leq U_{p^{\omega} G}(y)\right)$ there exists an automorphism (endomorphism) $\alpha$ of $G$ such that $\alpha(x)=y$. Corner constructed a fully transitive non-transitive $p$-group with countable first Ulm subgroup in [4] and it is a longstanding problem whether there exists a fully transitive non-transitive $p$-group with finite first Ulm subgroup. Partial results were obtained by Carroll and Goldsmith in [2], 3] and by Hennecke in [9], but a general solution hasn't been found yet. It is the aim of this paper to show that the answer to Corner's question is no for a large class of $p$-groups, namely the class of $p$-groups of type $A$. Here a $p$-group $G$

Received by the editors August 9, 2001 and, in revised form, September 27, 2001.

2000 Mathematics Subject Classification. Primary 20K01, 20K10, 20 K30.

The first author was supported by project No. G-0545-173,06/97 of the German-Israeli Foundation for Scientific Research $\&$ Development.

The second author was supported by the Graduiertenkolleg Theoretische und Experimentelle Methoden der Reinen Mathematik of Essen University.

(C)2002 American Mathematical Society 
is of type $A$ if $\operatorname{Aut}(G) \uparrow_{p^{\omega} G}=U\left(\operatorname{End}(G) \uparrow_{p^{\omega} G}\right)$. Our approach is ring theoretic and uses the structure of the Jacobson radical of a ring which acts (fully) transitively on a finite $p$-group.

We follow standard notation found in Fuchs [6] and Kaplansky [11]. Multiplication by an integer $n$ on some group $H$ is denoted by $n * i d \uparrow_{H}$ while applying two endomorphisms $\varphi$ and $\psi$ successively is denoted by $\varphi \psi$.

\section{A question by A. L. S. Corner}

All groups considered will be abelian $p$-groups, where $p$ is a prime. Let $G$ be such a group. For each ordinal $\alpha$ we define $p^{\alpha} G$ to be $p^{0} G=G$ and $p^{\alpha} G=\bigcap_{\beta<\alpha} p\left(p^{\beta} G\right)$. Then the height $|x|_{G}$ in $G$ of an element $x \in G$ is defined to be $\infty$ if $x \in p^{\alpha} G$ for all ordinals $\alpha$ and otherwise $\alpha$ if $x \in p^{\alpha} G \backslash p^{\alpha+1} G$, where infinity exceeds any ordinal. The Ulm sequence of an element $x \in G$ is given by $U_{G}(x)=\left(\alpha_{0}, \alpha_{1}, \alpha_{2}, \ldots\right)$ where $\alpha_{i}=\left|p^{i} x\right|_{G}$. The Ulm sequences are partially ordered by agreeing that $U_{G}(x) \leq U_{G}(y)$ if $\left|p^{i} x\right|_{G} \leq\left|p^{i} y\right|_{G}$ for all $i \geq 0$. By $G\left(U_{G}(x)\right)$ we denote the set of all elements $y \in G$ such that $U_{G}(y) \geq U_{G}(x)$.

Definition 1.1. Let $G$ be a $p$-group and $R$ a unital subring of $\operatorname{End}(G)$. We say that

(i) $R$ acts fully transitively on $G$ if for any $x, y \in G$ with $U_{G}(x) \leq U_{G}(y)$ there exists an element of $R$ which maps $x$ to $y$.

(ii) $R$ acts transitively on $G$ if for any $x, y \in G$ with $U_{G}(x)=U_{G}(y)$ there exists a unit of $R$ which maps $x$ to $y$.

(iii) $G$ is (fully) transitive if $\operatorname{End}(G)$ acts (fully) transitively on $G$.

We note that any direct summand of a fully transitive $p$-group $G$ is again fully transitive. Moreover, the question of deciding when a group is (fully) transitive is often made easier by the following fundamental observation of Corner 4 .

Lemma 1.2. A p-group $G$ is (fully) transitive if and only if $\operatorname{End}(G)$ acts (fully) transitively on the first Ulm subgroup $p^{\omega} G$ of $G$.

In the same paper [4] Corner showed that transitivity implies full transitivity if the first Ulm subgroup is a direct sum of cyclic groups of one and the same order $p^{n}$. We call such groups homogeneous. Motivated by this result it was shown in 4] that the converse does not hold. In particular Corner constructed an example of a $p$-group which is fully transitive but not transitive and $p^{\omega} G$ is homogeneous of cardinality $\aleph_{0}$. Thus the following question is natural:

Question 1.3. Is there a fully transitive non-transitive p-group with finite first Ulm subgroup?

If $G$ is a $p$-group, then $\operatorname{End}(G)\left\lceil_{p^{\omega} G}\right.$ is a subring $R$ of $\operatorname{End}\left(p^{\omega} G\right)$ and similarly $S=\operatorname{Aut}(G) \uparrow_{p^{\omega} G}$ is a subgroup of $\operatorname{Aut}\left(p^{\omega} G\right)$. Clearly $S \subseteq U(R)$, the units of $R$, but in general the inclusion may be strict. Hence Carroll and Goldsmith introduced the following notion in [3].

Definition 1.4. A p-group $G$ is said to be of type $A$ if

$$
\operatorname{Aut}(G) \uparrow_{p^{\omega} G}=U\left(\operatorname{End}(G) \uparrow_{p^{\omega} G}\right) .
$$


For instance any $p$-group with cyclic first Ulm subgroup is of type $A$ and all examples constructed in [4] and [13] are of type $A$. Nevertheless, examples of $p$ groups which are not of type $A$ may appear. The following result from [3] provides a helpful tool to handle $p$-groups of type $A$.

Lemma 1.5. Let $G$ be a p-group such that any unital subring of $\operatorname{End}(G)$ that acts fully transitively on $G$ also acts transitively on $G$. Then every fully transitive $p$ group $H$ of type A having $G$ as its first Ulm subgroup is also transitive.

The best results on Corner's Question 1.3 which are known up to now (to the authors' knowledge) are the following which can be found in [2, [3] and 9 .

Theorem 1.6. Let $G$ be a fully transitive p-group. Then $G$ is transitive if one of the following conditions holds:

(i) $p^{\omega} G=\mathbb{Z}\left(p^{n}\right) \oplus \mathbb{Z}\left(p^{n}\right)$ for some $n \in \mathbb{N}$.

(ii) $p^{\omega} G=\bigoplus_{i=1}^{n} \mathbb{Z}\left(p^{n_{i}}\right)$ for some $n, n_{i} \in \mathbb{N}$ such that $n_{i}<n_{j}$ if $i<j$.

\section{2. $p$-Groups With FINITE FIRST Ulm SUbGroup}

In this section we will mainly consider abelian $p$-groups $G$ of type $A$ with finite first Ulm subgroup, i.e., $p^{\omega} G=\bigoplus_{i=1}^{n} \mathbb{Z}\left(p^{m_{i}}\right)$ for some $m_{i}, n \in \mathbb{N}$. We show that for this class of groups, full transitivity implies transitivity. If $R$ is a ring, then we denote by $J(R)$ the Jacobson radical of $R$ and abbreviate $J(R)$ by $J$ if there is no danger of confusion.

Lemma 2.1. Let $n \in \mathbb{N}$ and $H=\bigoplus_{i=1}^{n} H_{i}$, where each $H_{i}$ is homogeneous of the form $\bigoplus_{j=1}^{k_{i}} \mathbb{Z}\left(p^{m_{i}}\right)$ for some fixed $m_{i} \in \mathbb{N}$ such that $m_{i}<m_{j}$ if $i<j$ and cardinals $k_{i}$. If $x, y \in H$ and $R$ is a unital subring of $\operatorname{End}(H)$ which acts (fully) transitively on $H$, then the following hold:

(i) If $n=1$, then:

(a) $\operatorname{ord}(x)=p^{k}$ if and only if $x \in p^{m_{1}-k} H \backslash p^{m_{1}-k+1} H$;

(b) $\operatorname{ord}(x) \leq \operatorname{ord}(y)$ if and only if $U_{H}(y) \leq U_{H}(x)$;

(c) if $\operatorname{ord}(x)=\operatorname{ord}(y)$, then $x \in J^{k} H$ if and only if $y \in J^{k} H$ for $k \in \mathbb{N}$.

(ii) If $U_{H}(x)=U_{H}(y)$, then $x \in J^{k} H$ if and only if $y \in J^{k} H$ for $k \in \mathbb{N}$.

(iii) $J H=H_{1} \oplus \cdots \oplus H_{n-1} \oplus p H_{n}$ if $H$ is finite, i.e. all $k_{i}$ are finite.

Proof. If $n=1$, then $H$ is homogeneous, hence the proof of $(a)$ is trivial. Moreover, if $x, y \in H$, then clearly $U_{H}(y) \leq U_{H}(x)$ implies $\operatorname{ord}(x) \leq \operatorname{ord}(y)$. Conversely, if $\operatorname{ord}(x) \leq \operatorname{ord}(y)$, then $|x| \leq|y|$ follows from (a). Thus $U_{H}(y) \leq U_{H}(x)$ using the fact that no Ulm sequence in $H$ has a gap before the first infinite entry. Therefore (b) holds.

To show (c), let $\operatorname{ord}(x)=\operatorname{ord}(y)$. Then (b) implies that $U_{H}(x)=U_{H}(y)$, hence it suffices to prove (ii). But (ii) is trivial since each $J^{k}$ is an ideal of $R$ and $R$ acts (fully) transitively on $H$. It remains to prove (iii). We have to show that $J H=H_{1} \oplus \cdots \oplus H_{n-1} \oplus p H_{n}$. Since $p * i d_{H} \in R$ is nilpotent, we obtain $p * i d_{H} \in J$ and hence $p H \subseteq J H$. Assume that there exists $x \in J H \backslash p H$ and write $x=\left(x_{1}, \cdots, x_{n}\right)$ with $x_{i} \in H_{i}$. Let $k$ be maximal such that $x_{k} \neq 0$ and $x_{k} \notin p H_{k}$. Then any element in $H_{1} \oplus \cdots \oplus H_{k}$ has Ulm sequence greater than or equal to 
$U_{H}(x)$, hence $H_{1} \oplus \cdots \oplus H_{k} \subseteq R x \subseteq R J H \subseteq J H$ by full transitivity. Therefore $J H=H_{1} \oplus \cdots H_{k-1} \oplus p H_{k} \oplus \cdots \oplus p H_{n}$ for some $1 \leq k \leq n$. Note that $H=J H$ would imply $H=0$ by Nakayama's Lemma ([1], Corollary 15.13). If $k<n$, then $H / J H=$ $\left(H_{k} \oplus \cdots \oplus H_{n}\right) /\left(p H_{k} \oplus \cdots \oplus p H_{n}\right)$ and we choose any element $x$ in $H_{k} \backslash J H$. It follows that $U_{H}(x)=\left(0,1, \cdots, m_{k}-1, \infty, \cdots\right)$ and obviously any element $z \in H \backslash J H$ satisfies $U_{H}(z) \leq U_{H}(x)$. Hence there exists an endomorphism $r \in R$ such that $r(z)=x$ and thus $R x \subseteq R z$. This proves that any $R / J$ submodule $K / J H$ of $H / J H$ contains the non-trivial module $(R x+J H) / J H$. But by Theorem XII.5 from [12] $H / J H$ decomposes as an $R / J$ module into a direct sum of simple $R / J$ modules, thus $H / J H=(R x+J H) / J H$. By Nakayama's Lemma ([1], Corollary 15.13) it follows that $R x=H$, a contradiction. Hence $k=n$ and $J H=H_{1} \oplus \cdots \oplus H_{n-1} \oplus p H_{n}$.

Next we will show that for the homogeneous case it is enough to consider groups of exponent $p$. Therefore we need the following proposition which enables us to consider $R / J$ acting on $H / J H(R x / J x$ for some $x \in H)$ instead of $R$ acting on $H$.

Proposition 2.2. Let $n \in \mathbb{N}$ and $H=\bigoplus_{i=1}^{n} H_{i}$, where each $H_{i}$ is of the form $\bigoplus_{j=1}^{k_{i}} \mathbb{Z}\left(p^{m_{i}}\right)$ for some fixed $m_{i} \in \mathbb{N}$ such that $m_{i}<m_{j}$ if $i<j$ and cardinals $k_{i}$. If $R$ is a unital subring of $\operatorname{End}(H)$, then the following hold:

(i) If $H$ is finite and $R$ acts (fully) transitively on $H$, then $R / J$ acts (fully) transitively on $H / J H$.

(ii) If $n=1$ and $R$ acts fully transitively on $H$ and $R / J$ acts transitively on $H / J H$, then $R$ acts transitively on $H$.

(iii) If $x, y \in H$ such that $U_{H}(x)=U_{H}(y)$, then $r(x)=y$ for some unit $r \in R$ if and only if $(s+J)(x+J x)=y+J x$ for some unit $(s+J) \in R / J$.

Here the action of $R / J$ on $H / J H(R x / J x)$ is given by $(r+J)(h+J H)=r(h)+J H$ $((r+J)(h+J x)=r(h)+J x)$.

Proof. First we prove (i) and assume that $H$ is as stated, hence finite. Let $R$ be a unital subring of $\operatorname{End}(H)$. Then it is easy to check that the action $(r+J)(h+J H)=$ $(r(h)+J H)$ of $R / J$ on $H / J H$ is well defined. Now assume that $R$ acts (fully) transitively on $H$ and let $\left(h_{1}+J H\right),\left(h_{2}+J H\right) \in H / J H$ be two non-zero elements of $H / J H$. Then $U\left(h_{1}+J H\right)=U\left(h_{2}+J H\right)$, since $J H=H_{1} \oplus \cdots \oplus H_{n-1} \oplus p H_{n}$ by Lemma 2.1 (iii) and hence $H / J H$ is a direct sum of copies of $\mathbb{Z}(p)$. Since $h_{1}, h_{2} \notin J H=H_{1} \oplus \cdots \oplus H_{n-1} \oplus p H_{n}$, we easily obtain $U\left(h_{1}\right)=U\left(h_{2}\right)$. Therefore there exists an (endomorphism) automorphism $r \in R$ such that $r\left(h_{1}\right)=h_{2}$. Thus $(r+J)\left(h_{1}+J H\right)=r\left(h_{1}\right)+J H=h_{2}+J H$ and $r+J$ is a non-trivial (endomorphism) automorphism of $R / J$ since otherwise $h_{2} \in J H$, a contradiction. Therefore $R / J$ acts (fully) transitively on $H / J H$.

To show (ii), let $n=1$ and assume that $R / J$ acts transitively on $H / J H$ with the action given as above and $R$ acts fully transitively on $H$. Let $h_{1}, h_{2}$ be two non-zero elements of $H$ such that $U\left(h_{1}\right)=U\left(h_{2}\right)$. We have to distinguish between two cases. Note that $h_{1} \in J H$ if and only if $h_{2} \in J H$ by Lemma 2.1 (ii):

Case 1: If $h_{1}, h_{2} \notin J H$, then $\left(h_{i}+J H\right) \neq 0$ for $i=1,2$, and hence $\operatorname{ord}\left(h_{1}\right)=$ $\operatorname{ord}\left(h_{2}\right)=p^{m}$ by Lemma 2.1 (i) and $U\left(h_{1}+J H\right)=U\left(h_{2}+J H\right)$. Therefore there exists an automorphism $\left(r_{0}+J\right) \in R / J$ such that $\left(r_{0}+J\right)\left(h_{1}+J H\right)=\left(h_{2}+J H\right)$. Since automorphisms lift modulo the Jacobson radical, $r_{0}$ is an automorphism of $H$ such that $r_{0}\left(h_{1}\right)-h_{2} \in J H$. Note that ord $\left(h_{1}\right)=p^{m}$ implies that $U\left(h_{1}\right) \leq U(x)$ for 
any $x \in H$ (Lemma 2.1 (i)(b)) and hence $R h_{1}=H$ since $R$ acts fully transitively on $H$. Thus $J H=J h_{1}$ and there exists $r_{1} \in J$ such that $r_{0}\left(h_{1}\right)-h_{2}=r_{1}\left(h_{1}\right)$. It follows that $\left(r_{0}-r_{1}\right)\left(h_{1}\right)=h_{2}$ and $\left(r_{0}-r_{1}\right) \in R^{*}$ since $\left(r_{0}-r_{1}\right) r_{0}^{-1}=1-r_{1} r_{0}^{-1} \in$ $1-J \subseteq R^{*}$.

Case 2: If $h_{1}, h_{2} \in J H$, then there exist $g_{1}, g_{2} \notin p H=J H$ such that $h_{i}=p^{m-k} g_{i}$ for some $k \in \mathbb{N}$ and $U_{H}\left(g_{1}\right)=U_{H}\left(g_{2}\right)$ for $i=1,2$. By Case 1 we obtain $r \in R^{*}$ such that $r\left(g_{1}\right)=g_{2}$, and hence $r\left(h_{1}\right)=h_{2}$.

Finally, (iii) is easy to check as above since units modulo the Jacobson radical lift and the action of $R / J$ on $R x / J x$ is well defined.

The following example shows that in Proposition 2.2 (ii) the assumption that $R$ acts fully transitively on $H$ cannot be avoided.

Example 2.3. Let $p>2$ be a prime, $H=\mathbb{Z}(p) \oplus \mathbb{Z}(p)$ and $R$ be the unital subring of $\operatorname{End}(H)$ consisting of all lower triangular $2 \times 2$-matrices over $\mathbb{Z}(p)$. Then $R$ acts neither fully transitively nor transitively on $H$ but $R / J$ acts (fully) transitively on $H / J H$.

Proof. Clearly no element of $R$ can map the element $(0,1)$ onto $(1,0)$, hence $R$ acts neither fully transitively nor transitively on $H$ since $(1,0)$ and $(0,1)$ have the same Ulm sequence. Moreover, it is easy to see that $J$ is the set of all $2 \times 2$-matrices over $\mathbb{Z}(p)$ which have a non-zero entry only in the left lower corner. Hence $J H=$ $\{(0, x): x \in \mathbb{Z}(p)\}$ and thus $H / J H \cong \mathbb{Z}(p)$. Now, if $(r, 0)+J H$ and $(s, 0)+J H$ are two non-zero elements in $H / J H$, then both elements have the same Ulm sequence and $\left(\begin{array}{cc}s r^{-1} & 0 \\ 0 & 1\end{array}\right)+J$ is an automorphism which maps $(r, 0)+J H$ onto $(s, 0)+J H$. Therefore $R / J$ acts (fully) transitively on $H / J H$.

By Proposition 2.2 it suffices to look at $R x / J x$ for some $x \in H$. For this we prove a more general theorem on finite semisimple rings.

Theorem 2.4. Let $1 \in R$ be a finite semisimple ring and $M$ a finitely generated $R$-module. If $u, v \in M$ and $r, s \in R$ such that $r u=v$ and $s v=u$, then there exists a unit $t \in R$ such that $t u=v$.

Proof. Since $R$ is semisimple it follows by the Artin-Wedderburn Theorem ([1], Theorem 13.6) that $R$ is the ring direct product $R \cong \prod_{i=1}^{k} \operatorname{Mat}_{n_{i}}\left(F_{i}\right)$ for some $k, n_{i} \in \mathbb{N}$ and some finite fields $F_{i}(1 \leq i \leq k)$. Since $M$ is finitely generated over $R$, it is projective ([12], Theorem XII.5 ). Hence [12], Theorem XII.4, implies that $M \cong \bigoplus_{i=1}^{l} R e_{i}$, where the $e_{i} \in R$ are minimal idempotents. Let $i \in\{1, \cdots, k\}$ and $E_{j}^{i} \in \operatorname{Mat}_{n_{i}}\left(F_{i}\right)$ be the matrix with 1 in the $(j, j)$ entry and zeros elsewhere $\left(j=1, \cdots, n_{i}\right)$. Then $E_{j}^{i}$ is a minimal idempotent in $\operatorname{Mat}_{n_{i}}\left(F_{i}\right)$ and it is easy to see that $\left\{E_{j}^{i}: j=1, \cdots, n_{i}\right\}$ is a complete set of minimal idempotents of $\operatorname{Mat}_{n_{i}}\left(F_{i}\right)$. Thus each $R e_{i}$ in the decomposition of $M$ is a copy of $F_{j}^{n_{j}}$ if $e_{i} \in \operatorname{Mat}_{n_{j}}\left(F_{j}\right)$. We let $M_{i}$ be the collection of all summands $\operatorname{Re}_{j}$ belonging to $\operatorname{Mat}_{n_{i}}\left(F_{i}\right)$, i.e., $M_{i}=\bigoplus_{e_{j} \in \operatorname{Mat}_{n_{i}}\left(F_{i}\right)} R e_{j}$. Then any $M_{i}$ is a direct sum of copies of $F_{i}^{n_{i}}$. Let $u, v \in M$ and $r, s \in R$ such that $r u=v$ and $s v=u$. We write $u=\left(u_{1}, \cdots, u_{k}\right)$ and $v=\left(v_{1}, \cdots, v_{k}\right)$ corresponding to the decomposition $M=\bigoplus_{i=1}^{k} M_{i}$. Moreover, 
we write $r=\left(r_{1}, \cdots, r_{k}\right)$ and $s=\left(s_{1}, \cdots, s_{k}\right)$ corresponding to the decomposition of $R=\prod_{i=1}^{k} \operatorname{Mat}_{n_{i}}\left(F_{i}\right)$. It follows that $r u=\left(r_{1} u_{1}, \cdots, r_{k} u_{k}\right)=v$ and $s v=\left(s_{1} v_{1}, \cdots, s_{k} v_{k}\right)=u$. Hence we obtain $r_{i} u_{i}=v_{i}$ and $s_{i} v_{i}=u_{i}$ for all $1 \leq i \leq k$. If we can find units $t_{i} \in \operatorname{Mat}_{n_{i}}\left(F_{i}\right)$ such that $t_{i} u_{i}=v_{i}$ for all $i$, then $t=\left(t_{1}, \cdots, t_{k}\right)$ would be a unit in $R$ mapping $u$ onto $v$ and we are done. Thus we may assume w.l.o.g. that $r, s \in R=\operatorname{Mat}_{n}(F)$ for some finite field $F$ and $u, v \in M \cong \bigoplus_{i=1}^{l} F^{n}$. Again we write $u=\left(u_{1}, \cdots, u_{l}\right)$ and $v=\left(v_{1}, \cdots, v_{l}\right)$ with $u_{i}, v_{i} \in F^{n}$ for all $1 \leq i \leq l$. Since $r$ and $s$ are matrices, they preserve linear dependence of vectors. Let $\left\{u_{i}: i \in I\right\}$ be a maximal linearly independent set among the $u_{i}$ 's. Then $r u_{i}=v_{i}$ and $s v_{i}=u_{i}$ implies that $\left\{v_{i}: i \in I\right\}$ is also maximal linearly independent among the $v_{i}$ 's. Obviously there is a non-singular matrix $t \in \operatorname{Mat}_{n}(F)$ mapping $u_{i}$ onto $v_{i}$ for all $i \in I$ since both sets $\left\{u_{i}: i \in I\right\}$ and $\left\{v_{i}: i \in I\right\}$ can be extended to bases of $F^{n}$. It follows immediately that $t u_{i}=v_{i}$ for all $1 \leq i \leq l$ and this completes the proof.

The following corollary contrasts Corner's example of a fully transitive nontransitive $p$-group with countable first Ulm subgroup from [4].

Corollary 2.5. Let $n \in \mathbb{N}$ and $H=\bigoplus_{i=1}^{n} \mathbb{Z}(p)$. If $R$ is a unital subring of $\operatorname{End}(H)$, then $R$ acts fully transitively on $H$ if and only if $R$ acts transitively on $H$.

Proof. By Lemma 2.1 (iii) we get that $J H=p H=0$ if $R$ acts (fully) transitively on $H$. Hence $J=0$ and $R$ is semisimple. Thus the result follows by Theorem 2.4 since $H$ is a finitely generated $R$-module. Note that transitivity implies full transitivity trivially in our situation since all non-zero elements have the same Ulm sequence.

Finally we obtain

Theorem 2.6. Let $H$ be a finite p-group. If $R$ is a unital subring of $\operatorname{End}(H)$ which acts fully transitively on $H$, then $R$ acts transitively on $H$. In particular every abelian p-group $G$ of type $A$ with finite first Ulm subgroup that is fully transitive must also be transitive.

Proof. Let $x, y \in H$ such that $U_{H}(x)=U_{H}(y)$. Then there exist $r, s \in R$ such that $r(x)=y$ and $s(y)=x$. If $r \in J$, then $x=s(r(x)) \in J x$, a contradiction, hence $r \notin J$ and similarly $s \notin J$. Thus $(r+J)(x+J x)=(y+J x)$ and $(s+J)(y+J x)=(x+J x)$ and by Theorem 2.4 it follows that $(t+J)(x+J x)=(y+J x)$ for some unit $(t+J) \in R / J$ since $R / J$ is finite semisimple. By Proposition 2.2 (iii) we get that $u(x)=y$ for some unit $u \in R$. The second claim follows by Lemma 1.5

\section{REFERENCES}

[1] F. Anderson and K. Fuller, Rings and categories of modules, Graduate Texts in Mathematics 13 (Springer, Berlin, 1992). MR 94i:16001

[2] D. Carroll, "Transitivity properties in abelian groups", doctoral thesis, Univ. Dublin, 1992.

[3] D. Carroll and B. Goldsmith, "On transitive and fully transitive abelian p-groups", Proc. of the Royal Irish Academy (1) 96A (1996) 33-41. MR 99f:20090

[4] A. L. S. Corner, "The independence of Kaplansky's notions of transitivity and full transitivity", Quart. J. Math. Oxford (2) 27 (1976) 15-20. MR 52:14090 
[5] S. Files and B. Goldsmith, "Transitive and fully transitive groups", Proc. Am. Math. Soc. 126 (1998) 1605-1610. MR 98g:20087

[6] L. Fuchs, Infinite Abelian Groups, Vol. I and II, (Academic Press, 1970 and 1973). MR 41:333, MR 50:2362

[7] B. Goldsmith, "On endomorphism rings of non-separable Abelian p-groups", J. of Algebra 127 (1989) 73-79. MR 91b:20077

[8] P. Griffith, "Transitive and fully transitive primary abelian groups", Pacific J. Math. 25 (1968) 249-254. MR 37:6374

[9] G. Hennecke, "Transitivitätseigenschaften abelscher $p$-Gruppen", doctoral thesis, Essen University, 1999.

[10] P. Hill, "On transitive and fully transitive primary groups", Proc. Amer. Math. Soc. 22 (1969) 414-417. MR 42:4630

[11] I. Kaplansky, Infinite abelian groups, (University of Michigan Press, Ann Arbor, 1954 and 1969). MR 16:444g MR 38:2208

[12] B. R. Mcdonald, Finite rings with identity, (Pure and Applied Mathematics, Dekker Inc., New York, 1974). MR 50:7245

[13] C. Megibben, "Large subgroups and small homomorphisms", Michigan Mathematical Journal 13 (1966) 153-160. MR 33:4135

[14] K. Shoda, "Uber die Automorphismen einer endlichen Abelschen Gruppe", Math. Ann. (1928) 674-686.

Department of Mathematics, University of the Philippines at Diliman, 1101 Quezon City, Philippines

E-mail address: agnes@math01.cs.upd.edu.ph

Fachbereich 6, Mathematik, University of Essen, 45117 Essen, Germany

E-mail address: lutz.struengmann@uni-essen.de 